\title{
México. Más Liberalismo, Menos Territorio: Transformaciones Regionales a la Luz de la Globalización
}

\author{
Javier Delgadillo Macías y \\ Felipe Torres Torres*
}

\begin{abstract}
It is recognized that the globalization generates distinguished changes in the organization from the territories where other factors of location are privileged, different from the traditional costs of transport and manual labor. Different authors affirm that these traditional factors no longer seem to be so determining in the evaluations of the companies' profitability: any territory can be good to develop several economic activities, since it is fulfilled certain requirements to improve the competitive conditions of the economic territorial units.

The task of measuring these changes and the form in which the regional processes of space integration or regional disparities associated to the new international scene, can be boarded from different methodologies for the regional analysis. In the present article one of them is exposed and accounted of the economic relations occurs that oppose to the present processes of development with the traditional forms of organization of the marginalized territories in Mexico.
\end{abstract}

Keywords: Regional Development, Globalization, Neoliberalism, Territorial Development.

Resumen: Se reconoce que la globalización genera cambios diferenciados en la organización de los territorios donde se privilegian otros factores de localización, distintos a los tradicionales costos de transporte y mano de obra. Autores diversos afirman que estos factores tradicionales ya no parecen ser $\tan$ determinantes en las evaluaciones de rentabilidad de las empresas: cualquier territorio puede ser bueno para desarrollar actividades económicas diversas, siempre que se cumpla con ciertos requisitos para mejorar las condiciones competitivas de las unidades económicas territoriales.

La tarea de medir estos cambios y la forma en que los procesos de integración espacial o las disparidades regionales asociadas al nuevo escenario internacional, pueden ser abordadas desde diferentes metodologías para el análisis regional. En el presente artículo se expone una de ellas y se da cuenta de las relaciones económicas que contraponen a los actuales procesos de desarrollo con las formas tradicionales de organización de los territorios marginados en México.

Palabras-clave: Desarrollo Regional, Globalización, Neoliberalismo, Desarrollo Territorial.

* Los autores son investigadores del Instituto de Investigaciones Económicas de la Universidad Nacional Autónoma de México - UNAM. E-mail: jdm@servidor.unam.mx. Recebido em 30/03/05 e aceito em 25/10/05. 


\section{Antecedentes}

Desde mediados del siglo XX la dinámica de la economía mexicana ya manifestaba una clara relación en términos de los intereses norteamericanos en nuestro país, tales como los procesos de inversión en la agricultura, la minería y el comercio binacional. Durante la segunda guerra mundial y hasta mediados de los sesenta se establecieron acuerdos migratorios que permitían la incorporación de mexicanos al campo y la industria estadounidense. Más recientemente, el desarrollo de maquiladoras, la ampliación de los intercambios comerciales y distintos programas de cooperación binacional, había intensificado las relaciones binacionales y transfronterizas.

Así, se puede constatar que incluso diez años antes del inicio formal del Tratado de Libre Comercio de América del Norte (TLCAN), que signaron México, Canadá y Estados Unidos, había iniciado un proceso de apertura de la economía y, por ello, se logró intensificar las relaciones económicas con los Estados Unidos. En 1986 México se incorporó formalmente al Acuerdo General de Aranceles y Comercio (GATT, por sus siglas en inglés), comenzando una progresiva disminución de sus cuotas arancelarias y entrando a procesos de desregulación financiera, energética y en el renglón de Inversiones foráneas. Previo al TLCAN también México ya había suscrito acuerdos comerciales con Costa Rica, Chile e Israel.

Sin embargo, lo que resulta significativo una década antes de iniciado el acuerdo comercial con Estados Unidos y Canadá fue la adopción de un modelo económico de rasgos exógenos que significó un cambio cualitativo en los procesos y espacios de acumulación de capital. Esto se constata a partir de la reorientación de los circuitos de inversión y los procesos de reestructuración industrial. La transición de un modelo territorial endógeno a uno de características exógenas derivó en la revaloración de los espacios no tradicionales y la emergencia de nuevas regiones y ciudades. En este nuevo esquema los puertos marítimos, las regiones fronterizas del norte y diversas ciudades de rango medio en el centro y norte del país se volvieron atractivas a las nuevas estrategias de expansión de los capitales foráneos en nuestro territorio.

Esta reactivación de regiones y ciudades, diferentes a los centros tradicionales, era posible por que representaban lugares donde se podrían implantar estrategias productivas compartidas con las empresas matrices en 
Estados Unidos o se utilizarían como espacios alternativos para establecer y/o ampliar las plataformas productivo-exportadoras. Este ha sido el caso de las ensambladoras automovilísticas, los distritos industriales de autopartes y los nuevos parques maquiladores de la electrónica, entre otros.

Un tema que fue discutido previamente a la entrada en vigor del Tratado de Libre Comercio de América del Norte fue el del posible impacto que éste tendría en términos de desarrollo urbano-regional en el territorio mexicano. A la luz de ello, se elaboraron escenarios sobre el impacto diferenciado del TLCAN en las distintas regiones y ciudades mexicanas. Se formularon modelos de competitividad regional y tendencias locacionales de sectores productivos; se llevaron a cabo ejercicios para determinar el grado de convergencia y polarización. Todo ello con la finalidad de conocer las debilidades y fortalezas de las entidades federativas y las ciudades, así como para tener una previsión sobre los posibles procesos de reestructuración urbano-regional bajo un contexto de mayor apertura económica. Entonces, diversos investigadores abordaron el debate sobre las regiones y sectores "ganadores" y "perdedores". Al respecto, se conocen al menos tres líneas de investigación que intentaron dar cuenta de estos problemas.

\section{El comportamiento regional de los indicadores}

La adopción del Tratado de Libre Comercio de América del Norte como propuesta de reactivación de la economía mexicana y la adaptación de ésta a los procesos de cambio económico mundial no contemplaron un mejoramiento específico de los desequilibrios regionales internos, si consideraron, como contraparte, un proceso dinámico de las actividades económicas que, de acuerdo con su inercia, generaría impactos territoriales en aquellas regiones que el modelo de economía protegida había marginado del desarrollo por fallas en la planeación y en la canalización de las inversiones gubernamentales.

Se adoptó a la inversión extranjera directa (IED) como el instrumento de reactivación pero sus efectos positivos habría que buscarlos, hasta ahora, en el nivel sectorial y no en el regional, aunque algunos estados del país presentan una relativa mejoría debido a la incorporación de determinados nichos territoriales a esta nueva dinámica de economía abierta.

De acuerdo con algunos analistas, la contribución de la IED a la economía 
mexicana ha sido compleja y ambigua. Se presenta como un factor importante para alcanzar una relativa estabilidad en algunas variables macroeconómicas y también como soporte a las estrategias de desarrollo iniciadas en 1988. En otro sentido genera una importante modernización de la economía por la vía de las empresas extranjeras asentadas en territorio nacional que han realizado diferentes tipos de asociaciones y fusiones con capital nacional. Sin embargo la IED no ha logrado resolver los principales retos de la economía mexicana: encadenamientos e integración productiva regional, empleo, financiamiento y sustentabilidad económica a mediano y largo plazo (DUSSEL, 2000).

Por tanto, desde una perspectiva macroeconómica, la IED es la principal fuente de financiamiento de la nueva estrategia de desarrollo y se ha convertido en uno de los más importantes factores que permiten la modernización económica y la orientación exportadora de un segmento del sector manufacturero. Previo al Tratado de Libre Comercio de América del Norte, el gobierno de México realizó modificaciones, reformas y leyes para sectores que una vez que el tratado entrara en operación, no se vieran afectados con los flujos de IED, sin embargo el TLCAN se ha convertido en el "techo" de gobierno para toda negociación bilateral o multilateral de comercio y de inversión, sobretodo si se toma en cuenta que anteriormente nuestro país no tenía acuerdos de inversión con otras naciones (DUSSEL, 2000).

Debido a que constituye uno de los factores para financiar el desarrollo de la economía mexicana y adecuarla a los niveles de competitividad que requiere la dinámica de una economía abierta, a partir de este Tratado el gobierno se convirtió en el principal promotor para desregular la inversión extranjera a nivel internacional y vincularla con el comercio. Lo anterior implica un alto grado de fragilidad para la economía mexicana, en la medida que se ve sujeta a las oscilaciones de la economía internacional y a un alto grado de dependencia de las crisis financieras de los países, o al nerviosismo de los capitales que tienen repercusiones inmediatas en las perspectivas del desarrollo local.

Entre 1994 y 1999 la IED presenta profundas oscilaciones como resultado de la crisis de 1994-1995 y la posterior incertidumbre internacional en torno a la crisis de Asia, Brasil y Rusia que presentaron efectos negativos para México hasta 1998. Alcanza su máximo histórico en 1994 con 14918 millones de dólares, cae $36.5 \%$ en 1995 , se recupera en 1997 y cae posteriormente como 
consecuencia de las crisis internacionales; presenta una tasa de incremento anual de $-9.1 \%$ durante $1994-1998$, en 1998 se ubica $31.6 \%$ por debajo de su nivel de 1994 (DUSSEL, 2000).

Sin embargo la IED continúa, hasta ahora, fuera de los márgenes de regulación estatal en cuanto a su destino regional.

Tanto las actividades de maquila como la reinversión de utilidades se han convertido en el segmento más dinámico ya que en 1998 representaron más del 38\% de la IED. Por si sola, la industria maquiladora ha generado el dinamismo del sector manufacturero, aumentando su participación de un 8.5\% del total en 1994 a $29.5 \%$ en 1998 .

Los subsectores productivos alimentos, bebidas y tabaco, productos metálicos, maquinaria y equipo, así como servicios financieros, seguros y cobranzas, se convirtieron en el principal destino de la IED. Entre la 10 principales ramas de mayor participación de la IED, seis se relacionan con el sector manufacturero y cuatro con servicios; el sector automotriz resulta el de mayo participación con 9.29\%. La IED se concentra en un número relativamente pequeño de ramas, diez de ellas concentraron durante el periodo 1994-1988 el 57.11\%. Estados Unidos es la principal fuente de origen con claras tendencias al incremento, ya durante el periodo 19941998 pasó de 41.8 a 55\%. En este periodo, la participación de Canadá y Estados Unidos juntos fue de 59.6\% y la Unión Europea alcanzó el 23.7\% (DUSSEL, 2000).

Vista por entidad federativa, la IED continúa lo mismo a la concentración en los estados más ricos, que hacia un relativo redireccionamiento hacia otras entidades. En el caso del D.F. disminuye de 7,566 millones de dólares (mdd) en 1994 a 3,384 en 1998, o bien del $78.6 \%$ de la IED total realizada al $66.4 \%$ para el mismo periodo. Nuevo León, Chihuahua, Baja California y el Estado de México se benefician de este proceso desde 1994 a través de la presencia de empresas maquiladoras, pero además se ha incorporado Jalisco dentro de una estrategia similar.

La mayoría de los análisis sobre inversión, realizados desde una base sectorizada pero que se abocan a ubicar sus principales impactos territoriales, concluyen ahora que éstas se dirigen a entidades que ofrecen la mayor rentabilidad y son ejecutadas a través de empresas con recursos suficientes para competir. Bajo tal supuesto, tenderán a localizarse y relocalizarse, según las condiciones de ajuste dadas por la creciente integración al mercado 
internacional. Esta cuestión puede ser oscilante en términos ascendentes o descendentes, pero mantiene una inercia positiva en cuanto a preferencia por los espacios de inversión regional ya prefigurados desde el ciclo de economía protegida.

Por ejemplo, el Distrito Federal ha continuado como el principal destino de la IED, le siguen los estados del Norte y Noreste, gracias a su mejor ubicación geográfica con respecto a los Estados Unidos, y la Región del Pacífico Norte (debido a la presencia de Jalisco y Sinaloa). Aunque el Distrito Federal y sus estados de influencia inmediata presentan ahora una caída relativa en términos de participación, que se traduce en ganancia para los estados del Norte, de todas maneras las condiciones no cambian hacia las regiones históricamente menos favorecidas del desarrollo.

En efecto, a partir de 1998 más de una cuarta parte de las empresas maquiladoras se localizaban ya en el interior del país, con una fuerte presencia en Yucatán, Guanajuato, mientras el Distrito Federal perdió participación, lo cual permite inferir un cambio en los patrones regionales de producción y empleo a partir de la implementación del TLCAN (KATZ, 2000).

También resulta claro el efecto territorialmente dispersor del Tratado de Libre Comercio de América del Norte que tiende a una relativa reconfiguración de las actividades económicas, pero ello está muy lejos de considerarse como el camino a un mejor equilibrio regional en el futuro, particularmente porque solo constituyen burbujas o nichos territoriales en regiones o estados del país donde tradicionalmente no había presencia de IED, pero esto no forma parte de una estrategia de planeación. No existen evidencias que permitan inferir su sostenimiento en el tiempo.

Sin embargo, dicho mecanismo, aún considerándolo como elemento incipiente, integra elementos nuevos. Durante la vigencia del modelo de economía protegida, que provocó una inercia concentradora de la actividad económica en el centro del país, con la apertura económica los patrones de localización se modificaron. En ello también influyó la caída del mercado interno a causa de la crisis de la economía, el impulso al cambio estructural a partir de una mayor competencia, el repliegue del estado en la economía, la desregulación de varios sectores, así como el incremento de inversiones externas y la reacción de grupos nacionales de empresarios que invirtieron para competir en el exterior. Todos estos factores disminuyeron el efecto que antes tenía la cercanía con los mercados locales nacionales como criterio de 
localización de las empresas y llevaron a una reorganización de los procesos en condiciones de competitividad internacional, sin embargo esto no cambió las distorsiones regionales y la permanencia de la brecha entre regiones ganadoras y perdedoras.

Un estudio realizado con información del periodo comprendido entre 1980 y 1999, concluye que en cuanto a concentración de la actividad económica el Distrito Federal registró la más alta en todos estos años, si bien no se mantuvo constante. Le siguieron el Estado de México, Nuevo León y Jalisco. Estas últimas entidades incluso incrementaron su participación según el criterio de venta de las empresas más grandes. A partir de 1986 y hasta 1990 aumentaron su participación Coahuila, Chihuahua, Sonora, Tamaulipas, y Baja California Norte; en el centro lo hicieron Guanajuato, San Luis Potosí y Tlaxcala, pero lo pierden Querétaro y Puebla; Veracruz y Sinaloa se mantienen sin variación. Ese comportamiento se presenta de igual manera entre 1991 y 1999 y se identifica con un patrón de desconcentración que coincide con la más baja participación del Distrito Federal. Sin embargo, en 1994 el Distrito Federal aumentó su participación junto con Nuevo León y Jalisco en detrimento del Estado de México; la participación de Nuevo León comenzó a ser mejor que la del Estado de México y la de Jalisco estaba muy cerca de alcanzarlo (CORONA, 2003).

Un efecto adicional tiene que ver con el patrón de distribución espacial de las actividades económicas. Considerando a las empresas más grande de México, éste presentó una desconcentración de 1980 a 1999 como consecuencia de la apertura de la economía y del menor dinamismo del mercado interno. Con la globalización se ha formado un conjunto de nodos, en especial de ciudades que responden a las exigencias del proceso de acumulación a escala mundial (CORONA, 2003). Pero esto no supone eliminar el problema de las desigualdades regionales, aunque se observa una relativa reconfiguración del territorio, dada por una presencia más dispersa de las empresas en función de las ventajas que han ofrecido algunos gobiernos estatales que, ante la falta de claridad de destino de las políticas económicas nacionales y su direccionamiento regional, fincan sus expectativas de mejoramiento económico en las oportunidades de inversión y desarrollo que podría ofrecer el TLCAN y otros países fuera de esta área de influencia.

Otro estudio realizado a través del comportamiento registrado por el PIB per cápita en el periodo 1993 - 1999, muestra un aumento en la desigualdad 
regional absoluta a partir de 1993, aunque la dispersión relativa no aumentó. Según este análisis los cambios estructurales a la economía parecen haber tenido dos efectos: a) en el sectorial, la tasa de crecimiento del sector manufacturero se ha incrementado sustancialmente a partir de 1993 y se expandió más que cualquier otro sector a excepción del transporte, almacenaje y comunicaciones. Por este efecto crecieron a mayores tasas los Estados donde la manufactura representa una elevada proporción de la producción; b) en lo regional, los Estados del norte han tenido un desempeño mejor al esperado, dada la estructura de su producción (MESSMACHER, 2000).

Lo anterior solo expresa las tendencias históricas del desequilibrio, junto con las limitaciones estructurales para frenarlo. En 1999 el PIB per cápita del Distrito Federal era seis veces superior al de los Estados más pobres, por ejemplo Oaxaca. Las regiones de elevado PIB per cápita son las que se localizan en la frontera norte del país, el Distrito Federal y el Estado de México, junto con Quintana Roo. Los Estados de ingresos medios se encuentran en la región norte, occidente y zona del Golfo, los Estados más pobres se ubican en la región sur del país.

Aunque no es evidente que las reformas estructurales a la economía realizados por el Estado mexicano, junto con el Tratado de Libre Comercio de América del Norte, hallan llevado a una dispersión mayor que la observada en los últimos 30 años, el hecho es que no se han reducido las diferencias regionales, según los indicadores positivos o de impacto que se analicen. El análisis de la evolución de las determinantes de largo plazo sugiere la posibilidad de un incremento en la desigualdad contrario a lo que las reformas estructurales perciben. Los Estados del norte presentan mejor desempeño, constituyendo una evidencia de los efectos regionales diferenciados, aunque esto no constituye una novedad, ni antes ni después de la implementación del TLCAN. Los estados del norte del país crecieron a tasas mayores que las esperadas mientras que los Estados de centro y sur el registro fue menor (MESSMACHER, 2000).

El criterio de la inversión como factor de desarrollo y competitividad de la economía mexicana en el contexto del TLCAN, parte del supuesto que las economías que basan su crecimiento en la inversión tienen más posibilidades de desarrollo en el largo plazo. Al producir excedentes existe la posibilidad de generar ahorro interno y externo; pero también supone inversiones en la innovación, en la medida en que no existe un solo país que halla logrado un 
alto desarrollo económico y social sin una estrategia basada en inversión previa en innovación y desarrollo tecnológico y son estos factores los determinantes en la competitividad en un mercado abierto.

El Plan Nacional de Desarrollo 2001-2006 plantea lograr el crecimiento con calidad con base en tres objetivos: a) promover una inserción ventajosa del país en el entorno internacional b) promover mayores flujos de inversión extranjera directa y c) fortalecer el mercado interno. Estos objetivos sin embargo, deberán alcanzarse en una situación de alta competencia regional mundial que va más allá de la innovación. Para China la inversión extranjera se ha logrado captar en los niveles actuales con base en el bajo costo de la mano de obra, estabilidad fiscal por 20 años y un costo cero del terreno, pero el potencial de integración a la economía regional local es limitada. En México funcionó el formato de empresa maquiladora; sin embargo el costo de la mano obra, así como la terminación del régimen para esta empresa ha provocado el desempleo y la migración, principalmente a países del Sureste asiático; el atractivo como destino de inversión en México se ha deteriorado (RUIZ, 2002).

Aquí es importante preguntarse también cómo satisfacer los requerimientos de la competencia externa, para no entrar en una fase de región rezagada en el escenario mundial, junto con una conciliación mínima de los indicadores intrarregionales para aprovechar más las ventajas internas y evitar los costos futuros de un mayor desequilibrio. Una primera aproximación es el conocimiento de los factores de distribución y su comportamiento y efectos más recientes, sobretodo a partir del TLCAN.

\section{Los nuevos saldos del Tratado de Libre Comercio de América del Norte (TLCAN)}

Tenemos claro que los impactos de una medida económica no pueden evaluarse solo a partir de un corte temporal, debido a que persisten diversos factores acumulados que son producto de estrategia anteriores y continúan influyendo en las inercias del desarrollo. De todas formas, dado que el TLCAN rompe con las inercias de la economía protegida pero, en términos de la apertura basada en el libre mercado no cuenta con formas de control de los procesos económicos internos, visualizamos sus primeros efectos regionales a partir de su implantación formal en 1994, según su impacto por entidad federativa, sin dejar de reconocer la influencia que los factores 
externos como la recesión de la economía norteamericana y otras crisis regionales de la economía mundial han tenido en la velocidad de los cambios positivos o negativos que prometía.

Así, un análisis de los datos hasta ahora expuestos pero ubicados en el periodo en que se cumplen los diez años del TLCAN y ocurre la liberalización más intensa de las barreras arancelarias, sobretodo en el sector agropecuario que a la vez repercute en el grueso de la estructura de México, no encontramos elementos sorprendentes de desempeño. Aumentan las exportaciones, sobretodo manufactureras y petroleras, aumenta la IED, y continua consolidándose el esquema de regiones ganadoras y perdedoras, solo con algunas ligeras variaciones, aunque también se presenta una fase de ascenso de las contradicciones y conflictos sociales.

El comportamiento del PIB por entidad federativa sigue en su fase de ascenso en los estados del Norte y del Centro, en este último caso debido a la presencia del D.F., en detrimento del Sur y con un ligero repunte del Sureste por la influencia de Yucatán y Quintana Roo, aunque todavía sin un impacto significativo.

Las tasas de crecimiento del PIB en los diez años del TLCAN, ubicaron al norte ya con una clara ventaja consolidando su dinámica ganadora; los comportamientos positivos en algunos estados fuera de esta región, no fueron suficientes para revertir esa tendencia, ni para reposicionar a las regiones perdedoras en los indicadores positivos de la apertura.

Durante el periodo aludido, Baja California, Coahuila, Chihuahua, Sonora, Tamaulipas y Nuevo León registraron las tasas de crecimiento más altas del PIB con más del 3\%, los sectores productivos que más contribuyeron a esta dinámica continúan siendo la industria manufacturera a lo cual se agregó, por la inercia derivada del crecimiento, el de los servicios; otros como el agropecuario y la construcción mantuvieron una contribución poco significativa.

En la misma dinámica de periodos anteriores al Tratado de Libre Comercio de América del Norte continuaron los Estados del centro, aunque con ligeras variaciones intrarregionales. El Distrito Federal observó una tendencia declinante al registrar una tasa de crecimiento de apenas $1.75 \%$, aunque sigue siendo la entidad que más contribuye en el escenario nacional, en mucho por el peso gravitacional y porque las medidas de desconcentración económica no han sido todo lo efectivas que se requiere, además de que continúa con los 
beneficios que acumuló durante el periodo de economía protegida.

Sin embargo otros estados del Centro observan ahora un repunte significativo, aunque no todo es producto delTLCAN, sino dela consolidación de inercias de localización productiva regional que ya venían ocurriendo de manera anticipada. Este es el caso de Querétaro que registró una tasa de crecimiento del 6.01\%, del Estado de México, de Puebla y Tlaxcala, lo que permitió que la región Centro no entrara en una fase de franco rezago.

La región Sureste logró un repunte con respecto a las condiciones que mantenía anteriormente, Yucatán creció a una tasa del 4.23\%, Tabasco 4.73\% y Quintana Roo al 3.61\% que resultan incluso superiores a algunos de los Estados del Norte, pero esto no implica un mejoramiento real del desarrollo regional. En un caso se debe a una fuerte presencia de empresas maquiladoras que se desplazaron del centro del país y que incluso han entrado ya a una fase migratoria, en el otro a la actividad petrolera que más bien presenta un carácter extractivo y no irradia beneficios y en el otro al renglón del turismo que observa la misma condición e incluso puede ser volátil e influir más en distorsiones regionales que en un mejoramiento sustentable a lo largo del tiempo.

Las entidades del Sur, junto con los Estados que históricamente han presentado esta condición, independientemente de su ubicación geográfica, tendieron a la misma condición de atraso e incluso manifestaron signos de empeoramiento. En esta condición encontramos los casos de Campeche, Colima, Chiapas, Hidalgo, Nayarit, Guerrero, Oaxaca y Veracruz, cuya tasa de crecimiento del PIB no se ubicó entre menos del 1\% y apenas arriba del $2 \%$, incluso en algunos años del periodo mostró un comportamiento negativo y en determinados sectores este comportamiento fue permanente. 


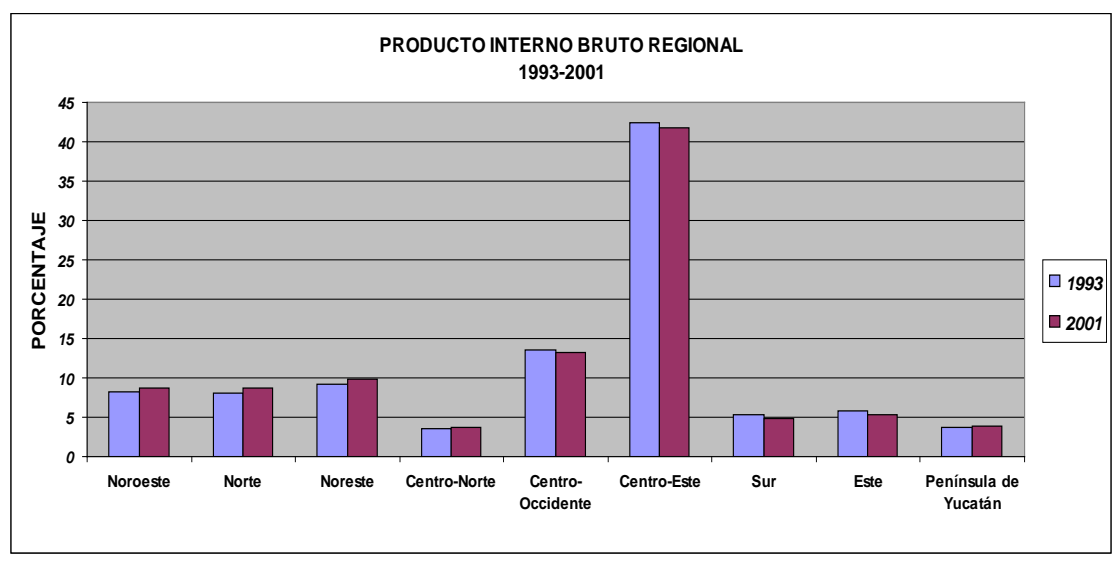

Gráfico 1 - Producto interno bruto regional 1993-2001

Por otra parte, también podemos detectar un grupo de entidades que no se ubican en una región determinada, ni necesariamente han correspondido a la categoría de regiones ganadoras o perdedoras, resultan más bien entidades cuyos gobiernos locales han mostrado una efectiva capacidad de promoción y gestión para atraer capitales y empresas principalmente maquiladoras. Este puede ser el caso de Guanajuato, Jalisco, Aguascalientes e incluso Colima; sin embargo debemos esperar un periodo más largo para determinar si consolidan su condición de regiones emergentes que han sabido aprovechar las ventajas que ofrece el TLCAN en cuanto a desarrollo.

De cualquier manera, el repunte de algunas entidades o la consolidación de ganadoras o perdedoras de otras que indiscutiblemente inciden en la reconfiguración de un mejor mapa económico del país, tampoco ha evitado el mejoramiento de los desequilibrios regionales.

A pesar de su declinación relativa en el crecimiento en el contexto nacional, con aproximadamente el 23\% de participación, el Distrito Federal sigue generando casi la cuarta parte de la riqueza del país, en detrimento de otras entidades que al no incentivar sus factores de crecimiento continúan en el atraso y como expulsoras permanentes de población.

El Distrito Federal junto con los Estados de México, Nuevo León, Jalisco, Veracruz y Puebla, concentraron más del 50\% del PIB nacional, donde la región Centro, si se excluye al Distrito Federal, Puebla y el Estado de México, queda en una condición de debilidad y solo presentaría su atractivo 
de amplio mercado de consumo; por esta razón, en el futuro, puede ocurrir una gran distorsión y desequilibrio intrarregional entre una ciudad central poderosa y unas entidades circundantes en condición de mayor atraso. Esta misma situación ocurre en el Norte con el caso de Nuevo León, aunque por el peso especifico que presenta cada estado dentro de la región en su conjunto ayuda a un equilibrio intrarregional más fuerte.

El resto de las entidades consideradas como emergentes presentan todavía un nivel de participación poco significativo. Por ejemplo Aguascalientes apenas si participa con el $0.97 \%$ de la riqueza, Guanajuato con el 3.36\%, Puebla con el 3.23\% y Querétaro con el 1.4\%, o sea, con todo y su dinamismo no genera ni la mitad de la riqueza que aporta el Distrito Federal. El resto de las entidades del Sur y Sureste ubicadas en condiciones de atraso, no igualaron juntas el valor generado por el Distrito Federal y el Estado de México.

En los diez años transcurridos del TLCAN, la IED ha sido determinante tanto para afianzar el avance del proyecto, como para reforzar el rango ganador, perdedor o emergente de las regiones. En este periodo se registró un flujo acumulado superior a 110 mil millones de dólares, sin embargo su distribución muestra de nuevo las inequidades regionales y determina ahora de manera más clara la tendencia estructural al desequilibrio.

Con todo y que presentó una abrupta declinación en los ańos posteriores inmediatos a la crisis de 1994, el Distrito Federal continúa como el principal receptor en este rubro; de un poco más de 7600 millones de dólares que recibió en 1994, en 1995 y 1996 disminuyó a menos de 5 mil millones de dólares, se recupera en 1997, cae de nuevo en 1998 y 1999 y en el 2001 capta una cifra récord de cerca de 19500 millones de dólares que correspondió a cerca del $80 \%$ de lo captado a nivel nacional.

El Distrito Federal mantiene una amplia jerarquía en cuanto a destino de la IED, que si bien canalizada en buena parte a ciertas actividades volátiles, de todas maneras genera una elevada distorsión e inhibe las perspectivas de atracción de otras entidades y regiones del país. Tan solo en 1999 que registra el nivel más bajo el Distrito Federal captó el 46.02\% de toda la IED que ingresó al país, en el 2001 repuntó al 76.89\% y en el 2002 bajó al 57\%. Aún así, el Distrito Federal prácticamente no bajó de un nivel de captación menor al $50 \%$ respecto al total nacional. 
El comportamiento de flujo regional de la IED se corresponde con el efecto al desequilibrio que inducen otros indicadores y que parecen agudizarse a partir del TLCAN, en tanto las estrategias de inversión derivadas están dirigidas hacia los servicios para mercados de consumo masivos y concentrados, o bien a sectores de la producción con un elevado componente tecnológico como el automotriz y algunas ramas manufactureras, los cuales ya tienen una localización bien definida con base a ventajas de infraestructura acumulada y afinada a lo largo del tiempo.

En esa condición aparece el Estado de México que por efecto de sus municipios concentrados en la ZMCM y aún resintiendo los efectos declinantes del Distrito Federal, de todas maneras participa entre el 3 y el $10 \%$ del total de las captaciones a nivel nacional. La región Norte muestra un comportamiento más compacto ya que los estados que la conforman registran los mayores niveles de IED. Nuevo León registró en el 2002 cerca del $10 \%$ en este rubro, mientras que Baja California participó con cerca del $8 \%$ en ese mismo ańo, Chihuahua el 6\%, Tamaulipas el 3.32\% y Sonora con el 1.51 por ciento.

Las entidades emergentes, por esa misma condición mantuvieron un repunte sorprendente. Por ejemplo, Guanajuato que en 1994 apenas si captó el $0.26 \%$ de la IED total nacional, en 1999 logró el $1.06 \%$ y en el 2002 el $1.27 \%$; en la misma condición encontramos a Jalisco que de un $0.60 \%$ captado en 1994, alcanzó el $6.64 \%$ en el 2000 y aunque se desplomó al $1.27 \%$ en el 2002, de todas maneras representó una participación altamente significativa en la dinámica del TLCAN. Para el resto de las entidades, la captación de IED resultó, además de comparativamente baja, muy errática, lo cual indica que de no tomarse otras medidas de atracción y de mejoramiento de infraestructura, es difícil que pueda resolver el problema del crecimiento y de los desequilibrios territoriales acumulados por esta vía.

Las tasas de crecimiento registradas en el periodo así parecen demostrarlo. Salvo Baja California, Chihuahua, Puebla, Sonora, Sonora, Tabasco, Chiapas, Campeche, Jalisco, Oaxaca y Nayarit que registraron tasas positivas en diferentes proporciones, en el resto, incluyendo el Distrito Federal, Estado de México y Nuevo León el saldo fue más bien negativo. Si bien es cierto que en mucho influyó la recesión de la economía norteamericana y sus efectos de arrastre sobre la economía mexicana, esta situación también es reflejo de la falta de competitividad total que ante el deterioro acumulado presenta ya 
una buena parte del territorio nacional, en la medida que una buena parte de los capitales se reorientaron hacia otras regiones mundiales como China el Sudeste Asiático y algunos países centroamericanos que ofrecen mejores atractivos fiscales y disminución de los costos de producción por la vía de menores salarios.

Un riesgo adicional es el origen mismo de la IED. A pesar que desde la década de los ochenta del Siglo pasado inicia el proceso de apertura formal de la economía mexicana, consolidada con la firma del TLCAN en 1994, lo cual generaría un proceso de diversificación de nuestras fuentes de financiamiento, en la práctica se han reforzado los lazos de dependencia con Estados Unidos en este rubro.

Si bien es cierto que se han firmado múltiples acuerdos comerciales con otros países, el incremento y participación proporcional de las estadounidenses, independientemente de su recesión, es notoria. En 1994 el 46.63\% de la IED provenía de los Estados Unidos; 6.96\% de Canadá: $5.58 \%$ del Reino Unido; $5.93 \%$ del Japón y 2.89\% de Alemania, para el año 2002 este esquema se había modificado radicalmente. En este año la IED directa proveniente de Estados Unidos, abarcó con 7071.40 de los 9696.40 millones de dólares ingresados al país por esta vía, el $72.93 \%$, donde cabe hacer notar que en el año inmediato anterior en que se registró la captación récord de 25334.40 millones de dólares, ese país concentró el $78.20 \%$ del monto total de las participaciones.

El resto de los países mostraron en cambio una tendencia descendente: Canadá, el otro socio del Tratado de Libre Comercio de América del Norte, con todo y sus ventajas de zona de influencia disminuyó su participación hasta caer al 0.33\%, el Reino Unido al 0.71\%, Japón al 0.99\%, Holanda al $5.01 \%$ y solo Alemania, Francia y España mejoraron pero con montos de participación todavía muy bajos.

En tal caso más que una apertura y diversificación de la economía mexicana por la vía del financiamiento, se trata de una franca integración a la economía de los Estados Unidos, pero además con efectos graves sobre el equilibrio regional, en la medida que los mayores montos se dirigen, o bien hacia a la Ciudad de México y la ZMCM, o a las entidades consolidas del Norte del país, sin mayor diversificación intrarregional. Además, los países emergentes en direccionamiento de inversiones hacia nuestro país, como 
sería el caso de España, Francia y Suiza, tampoco reflejan un mayor interés hacia la diversificación regional y sectorial.

Lo anterior tiende a reflejarse de manera poco positiva en la estructura económica del país. A pesar de que las exportaciones registraron durante el periodo una tasa de crecimiento del $11.08 \%$ y el de las importaciones de $8.8 \%$, de todas maneras seguimos dependiendo principalmente de las actividades petroleras y de algunas manufactureras sustentadas en empresas maquiladoras de alta volatilidad; tampoco se vislumbran posibilidades de que los Estados Unidos disminuya los niveles de participación como nuestro principal mercado de origen y destino. No contamos con datos precisos del origen regional en este rubro, pero es obvio que el grueso provienen de las regiones dinámicas que hemos venido analizando.

Es indiscutible el mejoramiento relativo que el TLCAN ha generado en el volumen de los flujos comerciales, en el incremento a la IED y en el mejoramiento de los niveles de competitividad debido a la presencia de empresas con inversiones sectoriales que requieren tecnología de punta, sin embargo ello no ha significado una relación favorable en los términos de intercambio. El balance del periodo es que la balanza comercial de México registró un saldo negativo de $-8.7 \%$, las regiones ganadoras avanzaron en su proceso de consolidación como tales y que las medidas compensatorias que hasta la fecha se han concebido desde la perspectiva gubernamental han resultado ineficaces para resolver el problema de los desequilibrios regionales que se siguen reflejando en el incremento de la pobreza, el deterioro ambiental de las regiones pobres y la descapitalización permanente tanto de su capital natural como humano sin nuevas opciones internas que lo reviertan, más allá del planteamiento de recuperar las dinámicas anteriores del mercado interno. 


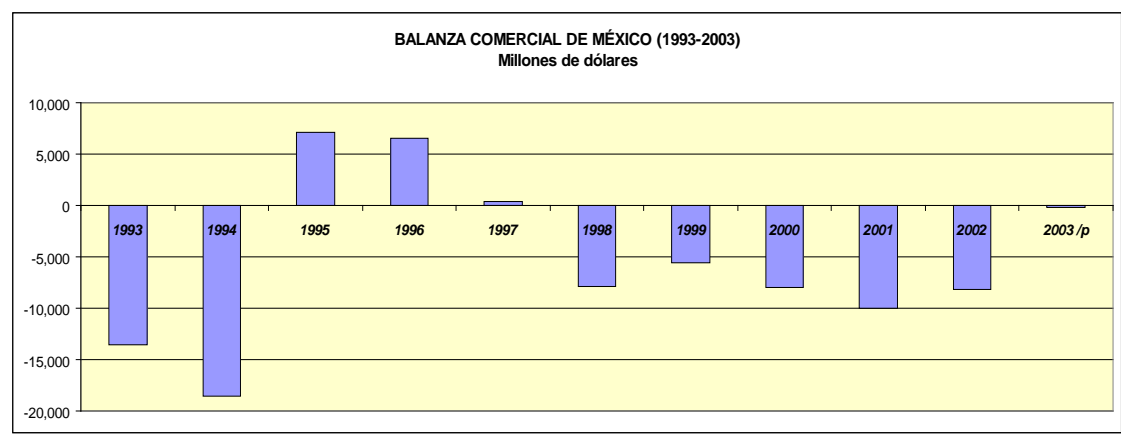

Gráfico 2 - Balanza comercial de México (1993-2003)

(Millones de dólares)

\section{Conclusiones}

Los desequilibrios regionales en México se han acentuado a lo largo del tiempo y son producto tanto de decisiones endógenas como exógenas que afectan en sentido positivo o negativo al territorio, las cuales finalmente expresan los procesos derivados de la política económica interna.

En el primer caso, durante el largo periodo, conocido en México como desarrollo estabilizador, la inversión controlada por una economía estatizada, se concentró en las principales ciudades, o bien se canalizó hacia macroproyectos sectoriales de infraestructura que no generaron procesos de crecimiento sostenido del entorno territorial, menos aún fueron capaces de traducir ese crecimiento en bienestar social, por lo que se fueron acentuando los desequilibrios regionales, junto con la pobreza y el abandono. Los programas de impulso industrial en las ciudades indujeron mecanismos de atracción de población que operaron en el mismo sentido, al concentrar inversión y población que desalentaron el crecimiento de las regiones más pobres, generaron fenómenos de macrocefalia urbana, junto con la manifestación de una crisis del sector agropecuario que opera con un claro desbalance de participación con respecto a otros sectores productivos.

En ese mismo esquema, las decisiones de inversión vinculadas a la política económica interna, permitió consolidar a las regiones que ya de por si presentaban ventajas en términos de dotación inicial de capital, lo que se complementó con la presencia de la mayor parte de obras de infraestructura 
para ganar una clara delantera con respecto al resto de las regiones, principalmente del Sur y Sureste que apenas si jugaron un papel marginal como proveedoras de materias primas, donde el desarrollo territorial registró un claro enfoque extractivo.

Por tanto, el Centro y el Norte se industrializaron, desarrollaron infraestructura para la atracción de capital productivo, principalmente mediante la inversión extranjera directa y con alto componente tecnológico, lo que finalmente sirvió como un gran soporte para los nuevos procesos que ya se avecinaban con la apertura comercial y la globalización de la economía mexicana.

Respecto de los procesos de globalización y la apertura expresadas en el Tratado de Libre Comercio de América del Norte, coincidieron con la implementación del modelo neoliberal, lo que en conjunto acentuó no solo la desigualdades regionales, sino también las sociales. Los desiguales términos de intercambio con dos de las principales potencias mundiales, junto con la orientación de la inversión hacia ramas productivas o nichos territoriales específicos de México, han generado ya un claro esquema de regiones ganadoras y perdedoras que obliga a repensar el futuro de nuestro país en la dimensión de las dinámicas mundiales, para contrarrestar el abandono y la pobreza específica de esas regiones, pero que afecta al conjunto del territorio nacional. 


\section{Referencias Bibliográficas}

AGUILAR, Ismael. Monterrey: formas de integración a la economía del sur de Estados Unidos. Comercio Exterior, México, v. 45, n. 5, mayo. 1995.

BAIGORRI, Artemio; CORTÉS, Georgina. Un nuevo desafío en el análisis regional: las ciudades y regiones fronterizas. Ponencia presentada en la XXIII Reunión Española de Estudios Regionales, Valencia, España, 1997.

BATALLA, Angel Bassols. Investigaciones urbanas y regionales de México: ¿Para conocer o transformar una realidad? En: MUNOZ, Humberto (Coord.). La sociedad mexicana frente al tercer milenio. México: Edit. Miguel Ángel Porrúa y Coordinación de Humanidades, UNAM, 1999.

CHAMBOUX-LEROUX, Jean Ives. Efectos de la apertura comercial en las regiones y en la localización industrial en México. Revista Comercio Exterior, México, v. 51, n. 7, julio. 2001.

CORONA, J. M. Ángel. Efectos de la Globalización en la distribución espacial de las actividades económicas. Revista Comercio Exterior, México, v. 53, n. 1. 2003.

DUSSEL, Enrique. La inversión extranjera en México. Serie Desarrollo Productivo, Santiago de Chile, CEPAL, ECLAC, n. 80, 2000.

GARCÍA, Francisco Arroyo. Dinámica del PIB de las entidades federativas en México, 1980-1999. Comercio Exterior, México, v. 51, n. 7, julio. 2001.

GONZÁLEZ, Pablo Wong. Integración de América del Norte: implicaciones para la competitividad y competencia internacional de las regiones. Santiago de Chile: Dirección de Políticas y Planificación Regionales, ILPES, docto. 97/17, 1997.

KATZ, Isaac. Impacto regional del TLCAN. Un análisis de la industria manufacturera. En: LEYCEGUI, Beatriz; CASTRO, Rafael Fernández de (Coord.) ¿Socios naturales? Cinco años del Tratado de Libre Comercio de América del Norte - TLCAN. México: Distrito Federal, ITAM, 2000. 
LEYCEGUI, Beatriz. Acordar para disentir: la solución de controversias en el Tratado de Libre Comercio de América del Norte. En: LEYCEGUI, Beatriz; CASTRO, Rafael Fernández de (Coord.) ¿Socios naturales? Cinco años del Tratado de Libre Comercio de América del Norte - TLCAN. México: Distrito Federal, ITAM, 2000.

LINARTAS, Miguel Messmacher. Desigualdad regional en México. El efecto del TLCAN y otras reformas estructurales. Documento de Investigación n. 2000-4, Dirección General de Información Económica del Banco de México, 2000.

MACÍAS, Javier Delgadillo et al. El desarrollo regional de México en el vértice de dos milenios. México: Edit. Miguel Ángel Porrúa e Instituto de Investigaciones Económicas, UNAM, 2001.

MARTÍNEZ, Carlos González. Los senderos de la integración: experiencias de España y México. Comercio Exterior, México, v. 45, n. 2, 1995.

OLAZÁBAL, Tito Alegría. Reestructuración productiva y cambio territorial: un segundo eje de industrialización en el norte de México. Revista de la Cepal, Santiago de Chile, n. 61, p. 187-204, abril. 1997.

OLAZÁBAL, Tito Alegría. Juntos pero no revueltos: ciudades en la frontera México-Estados Unidos. Revista Mexicana de Sociología, México, Instituto de Investigaciones Sociales, UNAM, v. 62, n. 2, abril-junio. 2000.

POLÈSE, Mario y Salvador Pérez Mendoza. La integración económica norteamericana y cambio regional en México. Comercio Exterior, México, v. 45, n. 2, 1992.

PRESIDENCIA DE LA REPÚBLICA. Plan Puebla-Panamá. Documento base, 2001.

REINOSO, Eduardo. La competitividad de los estados mexicanos. México: Centro de Estudios Estratégicos del Instituto Tecnológico de Estudios Superiores Monterrey, 1995.

ROSAS, María Cristina. México y Canadá ante los procesos de la globalización y regionalización. En: IBARRA, Guillermo; RUELAS, Ana Luz (Coords.). México y Canadá ante los procesos de la globalización. Fronteras tensionadas en América del Norte. México: Grupo Editorial Porrúa, Universidad Autónoma de Sinaloa, 2000. 
RÓZGA, Ryzard. La polarización espacial en las teorías del desarrollo regional. Revista Gestión y Política, México, Distrito Federal, CIDE, v. 111, n. 1, 1994.

RUIZ, Rocío. Política económica de la competitividad. Revista El Mercado de Valores, México, Distrito Federal, n. 12, año LXII, 2002.

SCHNEIDER, Julie. ElTLC y el transporte: impactos en la frontera MéxicoEstados Unidos. Borderlines, v. 8, n. 5, junio. 2000.

SEDESOL. Programa de 100 ciudades. Tratado de Libre Comercio y Estrategian de apoyo al desarrollo urbano. México: Dirección General de Desarrollo Urbano, 1994.

VILLARREAL, Gustavo Garza. Desconcentración espacial, tecnología y localización industrial en México. El conjunto de parques y ciudades industriales 1953-1988. México: El Colegio de México, 1991.

ZAMORA, José Gasca. Espacios trasnacionales. Interacción, integración y fragmentación en la frontera México-Estados Unidos. México: Edit. Miguel Ángel Porrúa, Instituto de Investigaciones Económicas, 2000. 